\title{
Linear Aglycones Are the Substrates for Glycosyltransferase DesVII in Methymycin Biosynthesis: Analysis and Implications
}

\author{
Chai-Lin Kao, Svetlana A. Borisova, Hak Joong Kim, and Hung-wen Liu * \\ Division of Medicinal Chemistry, College of Pharmacy, and Department of Chemistry and \\ Biochemistry, University of Texas, Austin, Texas 78712
}

\begin{abstract}
Macrolides are an important class of antibiotics used to treat infections caused by Grampositive pathogens. This group of compounds consists of two essential structural components: a polyketide aglycone and the appended sugars. The aglycone is biosynthesized by the sequential condensation of acyl thioesters catalyzed by polyketide synthases (PKS). ${ }^{1}$ The PKS genes (pikAI to pikAIV) in the pik gene cluster from Streptomyces venezuelae, which produces methymycin (1a) and neomethymycin (1) $)$, represent a well characterized system. ${ }^{2}$ The polyketide chain is made starting from $\mathbf{2}$ by PikAI, and passed on to the subsequent modules in an assembly line fashion (Scheme 1). The mature polyketide chain $(\mathbf{3})$ is released in the cyclic form (4) from PKS by the action of a thioesterase (TE) domain located at the $C$-terminus of the last module (module 6). This 12-membered ring macrolactone (4) is the precursor of methymycin (1a) and neomethymycin $(\mathbf{1 b}) .^{3}$
\end{abstract}

Advances in our understanding of polyketide biosynthesis have enabled the recombination of the PKS genes to construct a diverse array of new ring systems. ${ }^{1 \mathrm{c}, 1 \mathrm{e}, 4}$ Without the sugar components, however, these new compounds are usually biologically inactive. It is generally believed that glycosylation by the action of dedicated glycosyltransferases occurs after the cyclic aglycone (such as 4) is released from the PKS. However, the failure of in vitro demonstration of macrolide glycosyltransferase activity in the presence of a necessary TDPsugar donor and aglycone acceptor raised questions about the glycosylation step. The situation was clarified somewhat by the recent discovery of an auxiliary protein (DesVIII), which activates the glycosyltransferase (DesVII) responsible for the attachment of TDP-desosamine (7) to $4 .{ }^{5}$ This unusual property of DesVII may account for the fact that activity has not been previously verified by conventional assays. ${ }^{6}$ However, whether the acyl carrier protein (ACP)bound linear polyketide chain (3) can also be processed by the corresponding glycosyltransferase in addition to the cyclic aglycone has never been explored. Reported herein are the results of our investigation indicating that the linear polyketide chain $\mathbf{8}$ (an analogue of 3) can also serve as a chemically competent substrate for the macrolide glycosyltransferase DesVII.

As depicted in Scheme 2, the synthesis of the linear substrate $\mathbf{8}$ from 4, which was produced by $S$. venezuelae mutant KdesI-80, ${ }^{7}$ was initiated by reduction of the C-7 keto group $(\mathbf{4} \rightarrow$ 9), followed by protection of the $\mathrm{C}-3$ and $\mathrm{C}-7$ hydroxyl groups $(\mathbf{9} \rightarrow \mathbf{1 0})$, and oxidative cleavage of the double bond between $\mathrm{C}-8$ and $\mathrm{C}-9$ of $\mathbf{1 0}$ to give 11. ${ }^{8}$ The sulfone derivative of the sixcarbon $\left(\mathrm{C}_{9}-\mathrm{C}_{13}\right)$ fragment 12 was prepared using 13 as the chiral building block, which was reacted with propionaldehyde to yield the syn aldol product 14 . Subsequent reactions involving silyl protection $(\mathbf{1 4} \rightarrow \mathbf{1 5})$, reduction with $\mathrm{NaBH}_{4}(\mathbf{1 5} \rightarrow \mathbf{1 6})$, Mitsunobu reaction with 1phenyl- $1 H$-tetrazole-5-thiol $(\mathbf{1 6} \rightarrow \mathbf{1 7})$, and oxidation using a molybdate catalyst, ${ }^{9}$ led to the sulfone 12. This fragment (12) was then coupled to 11 under the Julia-Kocieñski olefination

E-mail: h.w.liu@mail.utexas.edu. 
conditions. 9,10 Interestingly, the anticipated diene compound was produced only in a trace amount. Instead, the hydrolyzed acid $\mathbf{1 8}$ was obtained in $50 \%$ yield. ${ }^{11}$ Consecutive deprotection of $\mathbf{1 8}$ gave $\mathbf{1 9}$ in $\mathbf{7 2 \%}$ yield. Due to the difficulty of preparing the ACP-bound linear polyketide chain (3), the intended linear substrate was synthesized as the $\mathrm{N}$ acetylcysteamine (NAC) thioester via esterification of 19 with $N$-acetyl-cysteamine. Selective oxidation of $\mathbf{2 0}$ afforded the desired product $\mathbf{8}$ in $82 \%$ yield. $^{12}$

Incubation of 8 with TDP-D-desosamine (7) in the presence of DesVII and DesVIII resulted in the formation of a more polar compound. ${ }^{13}$ This compound was purified by HPLC and the high-resolution CI-MS mass (calc. for $\mathrm{C}_{29} \mathrm{H}_{53} \mathrm{~N}_{2} \mathrm{O}_{7} \mathrm{~S}[\mathrm{M}+\mathrm{H}]^{+}$573.3573, found 573.3566) is consistent with the anticipated glycosylated product 21 (Scheme 3). The 7-OH compound 20 was also tested for chemical competence as a substrate for DesVII/DesVIII. Two new compounds were isolated from this incubation mixture. The major product is singly glycosylated as indicated by the high resolution CI-MS mass (calc. for $\mathrm{C}_{29} \mathrm{H}_{55} \mathrm{~N}_{2} \mathrm{O}_{7} \mathrm{~S}[\mathrm{M}$ $+\mathrm{H}]^{+} 575.3730$, found 575.3729). The predicted structure of this product is $\mathbf{2 2}$. The minor, and less polar product has a molecular mass corresponding to the dehydrated 22 (CI-MS calc. for $\mathrm{C}_{29} \mathrm{H}_{53} \mathrm{~N}_{2} \mathrm{O}_{6} \mathrm{~S}[\mathrm{M}+\mathrm{H}]^{+}$557.3624, found 557.3618). The dehydration is unlikely enzymecatalyzed, but an artifact of the incubation ( $\mathrm{pH} 8$ ) or isolation conditions. HPLC analysis of the incubation mixtures enabled the estimation of a 12\% yield for $\mathbf{2 1}$ from $\mathbf{8}$ and a $9 \%$ yield for $\mathbf{2 2}$ and the dehydrated product from $\mathbf{2 0}$. The low yields of the products precluded a thorough characterization of the structures, but a single product formation in all cases suggests that these compounds are glycosylated at $3-\mathrm{OH}$ group. ${ }^{14}$

For comparison, glycosylation of the cyclic aglycone $\mathbf{4}$ and the C-7 reduced form 9 were also examined under the same conditions. Again, a single glycosylated product was isolated in each case. NMR analysis confirmed the structure of the product resulting from $\mathbf{9}$ to be $\mathbf{2 3}$ (obtained in $45 \%$ yield), with the correct MS mass (calc. for $\mathrm{C}_{25} \mathrm{H}_{46} \mathrm{NO}_{6}[\mathrm{M}+\mathrm{H}]^{+} 456.3325$, found 456.3327). As expected, compound 5 was obtained from the incubation with 4 in nearly quantitative yield (CI-MS calc. for $\mathrm{C}_{25} \mathrm{H}_{44} \mathrm{NO}_{6}[\mathrm{M}+\mathrm{H}]^{+} 454.3169$, found 454.3173). ${ }^{15}$ Clearly, the cyclic forms are much better substrates: the yield of glycosylation is 5-fold higher for $\mathbf{9}$ versus $\mathbf{2 0}$ and at least 8 -fold more for $\mathbf{4}$ versus $\mathbf{8}$. The analysis also shows that DesVII prefers the C-7 keto group over the C-7 OH group ( 8 versus 20 , and 4 versus 9 ).

In summary, this study demonstrated for the first time that a macrolide glycosyltransferase can also recognize and process the linear precursor of its macrolactone substrate with reduced, but measurable activity. The reaction is regioselective because only one singly glycosylated product is generated in each case despite the presence of multiple potential glycosylation sites. This finding is significant for three reasons. First, a similar capability is possible for other macrolide glycosyltransferases, and the glycosylation of the linear polyketide may be a minor glycosylation pathway during the biosynthesis of macrolide antibiotics. Second, the substrate flexibility of macrolide glycosyltransferases is apparently extended to linear aglycones as well as cyclic analogues. This property expands the opportunities for glycodiversification of secondary metabolites. More importantly, this study implicates that the substitution patterns nearby the glycosylation site, and not the ring conformation, are the major recognition determinants for glycosyltransferases. ${ }^{16}$ This finding sets the stage for a future investigation to establish the minimal recognition elements for this class of glycosyltransferases. However, the fact that $\mathbf{8}$ and $\mathbf{4}$ are better substrates (than $\mathbf{2 0}$ and $\mathbf{9}$, respectively) indicates that the specificity of DesVII is also sensitive to the substituents distant from the glycosylation site. It has been shown that $\mathbf{8}$ can be cyclized to $\mathbf{4}$ by pik-TE in vitro. ${ }^{12}$ It remains to be tested if the thioesterase is also capable of cyclizing the glycosylated linear polyketides. 


\section{Supplementary Material}

Refer to Web version on PubMed Central for supplementary material.

\section{Acknowledgements}

This work was supported by the National Institutes of Health Grant GM54346.

\section{References}

1. For reviews, see: (a) Rawlings BJ. Nat Prod Rep 2001;18:190-227. [PubMed: 11336289](b) RawlingsBJibid231281 (c) Staunton J, Weissman KJ. Nat Prod Rep 2001;18:380-416. [PubMed: 11548049](d)WalshCAntibiotics: Actions, Origins, Resistance, edASM PressWashington, D.C2003 (e) Weissman KJ. Phil Trans R Soc Lond A 2004;362:2671-2690.

2. Xue Y, Zhao L, Liu H-w, Sherman DH. Proc Natl Acad Sci USA 1998;95:12111-12116. [PubMed: 9770448]

3. Aglycone 4 is produced under the growth conditions where the ketosynthase $\left(\mathrm{KS}_{6}\right)$ domain of module 6 is expressed in a truncated form. Under conditions when a full length $\mathrm{KS}_{6}$ is expressed, the released cyclic product is the 14-membered ring narbonolide, which is the precursor for narbomycin and pikromycin. [ (a) Xue Y, Sherman DH. Nature 2000;403:571-575. [PubMed: 10676969] (b) Beck BJ, Yoon YJ, Reynolds KA, Sherman DH. Chem Biol 2002;9:575-583. [PubMed: 12031664]

4. For reviews, see: (a) Strohl WR. Metab Eng 2001;3:4-14. [PubMed: 11162228] (b) Rodriguez E, McDaniel R. Curr Opin Microbiol 2001;4:526-534. [PubMed: 11587928] (c) Walsh CT. Chembiochem 2002;3:125-134. [PubMed: 11921390]

5. Borisova SA, Zhao L, Melancon CEI, Kao CL, Liu H-w. J Am Chem Soc 2004;126:6534-6535. [PubMed: 15161264]

6. A similar requirement has since been observed for other glycosyltransferases involved in the biosynthesis of macrolides [ (a) Melancon CE 3rd, Takahashi H, Liu HW. J Am Chem Soc 2004;126:16726-16727. [PubMed: 15612702] (b) Yuan Y, Chung HS, Leimkuhler C, Walsh CT, Kahne D, Walker S. J Am Chem Soc 2005;127:14128-14129. [PubMed: 16218575]] and anthracyclines [ (c) Lu W, Leimkuhler C, Gatto GJJ, Kruger RG, Oberthur M, Kahne D, Walsh CT. Chem Biol 2005;12:527-534. [PubMed: 15911373]] Preliminary data suggested that DesVIII and its homologues do not directly participate in the coupling reaction, but may serve as chaperones to activate the respective glycosyltransferase [see ref $6 \mathrm{~b}$ and (d) Borisova SA, Zhang C, Takahashi H, Zhang H, Wong AW, Thorson JS, Liu H-w. Angew Chem Int Ed. in press].

7. Borisova SA, Zhao L, Sherman DH, Liu H-w. Org Lett 1999;1:133-136. [PubMed: 10822548]

8. Attempts to cleave the internal double bond using $\mathrm{OsO}_{4} / \mathrm{NaIO}_{4}$ were unsuccessful, possibly due to the migration of the acetyl group from the C-7 hydroxyl to C-8 hydroxyl of the diol intermediate. Ozonolysis yielded the acyclic dialdehyde 11 without complications.

9. Paquette LA, Chang SK. Org Lett 2005;7:3111-3114. [PubMed: 15987218]

10. Blakemore PR, Cole WJ, Kocienski PJ, Morley A. Synlett 1998:26-28.

11. Such a transformation could occur by a $\beta$-fragmentation facilitated by the oxyanion generated after the addition of 12 to 11 .

12. A more efficient synthesis of 8 based on direct hydrolysis to open the macrolactone ring of 4 was recently reported [ (a) Aldrich CC, Beck BJ, Fecik RA, Sherman DH. J Am Chem Soc 2005;127:8441-8452. [PubMed: 15941278] (b) Aldrich CC, Venkatraman L, Sherman DH, Fecik RA. J Am Chem Soc 2005;127:8910-8911. [PubMed: 15969542]]. However, the hydrolysis was slow and two C-2 stereoisomers were generated during hydrolytic ring opening, which had to be separated by HPLC.

13. See Supporting Information for details.

14. However, glycosylation at $11-\mathrm{OH}$ remains a possibility.

15. Since neither 9 nor 23 possess a chromophore, an HPLC instrument equipped with the universal Corona charged aerosol detector (CAD) was used to show the conversion of 9 to 23 as the only product.

16. This is in agreement with our previous observation of aglycone specificity for DesVII (see ref. 6d). 


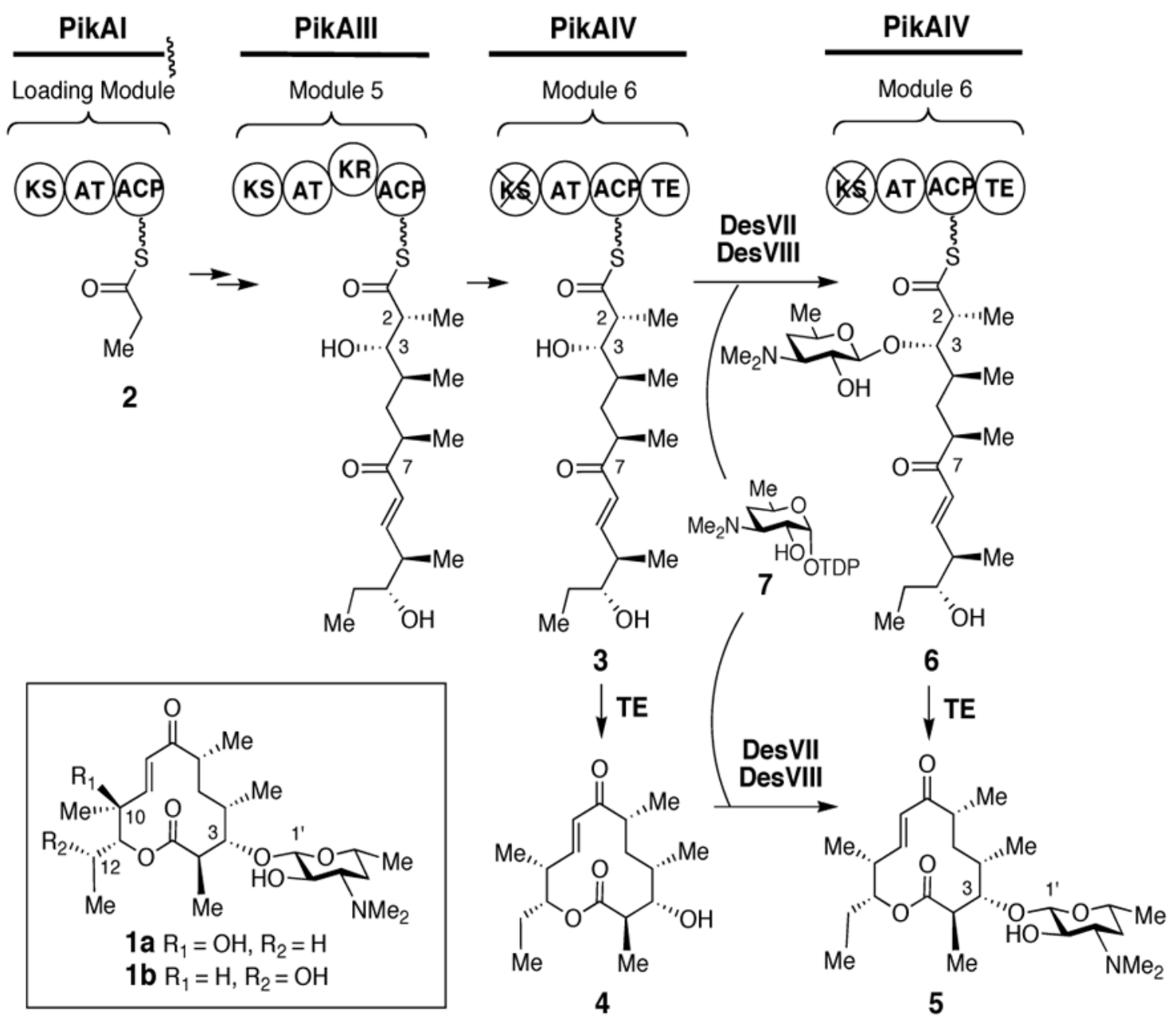

Scheme 1. 


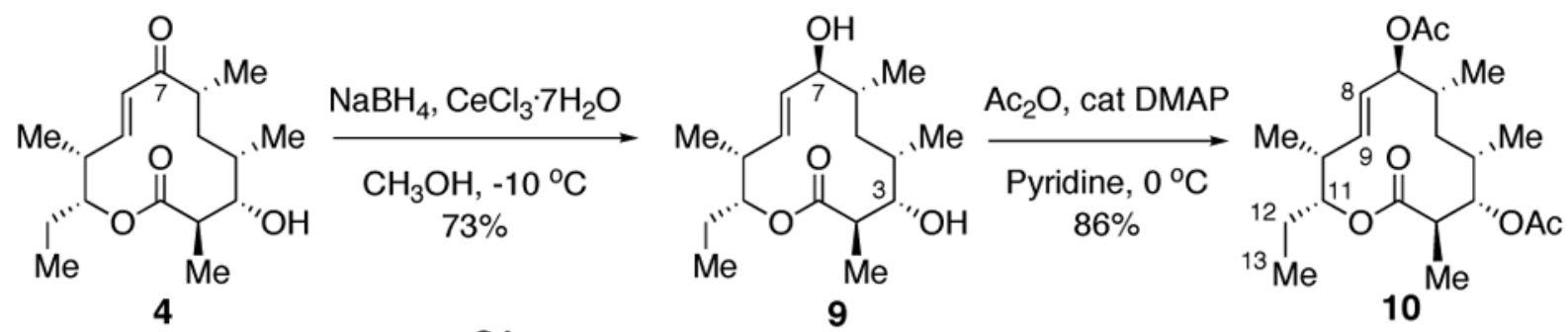

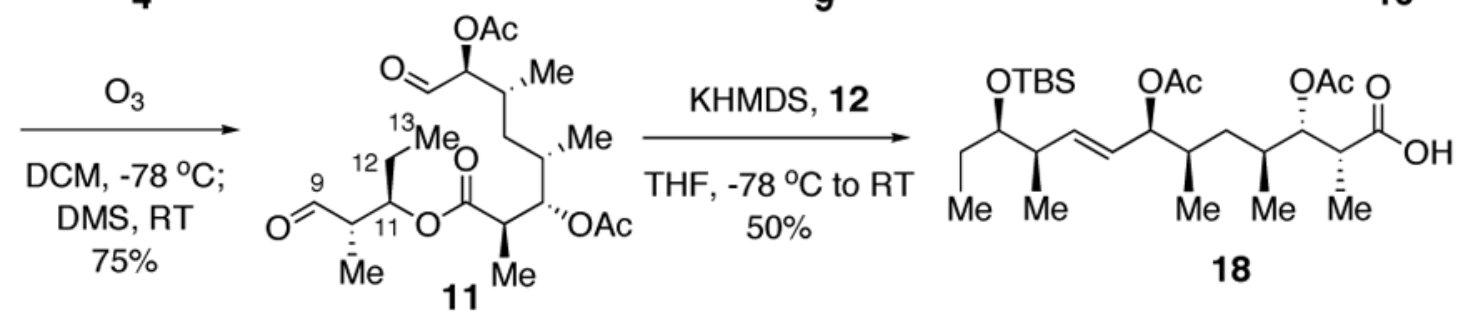

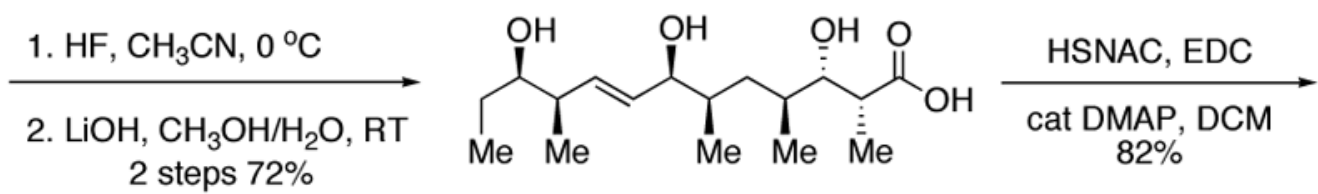
2 steps $72 \%$

19

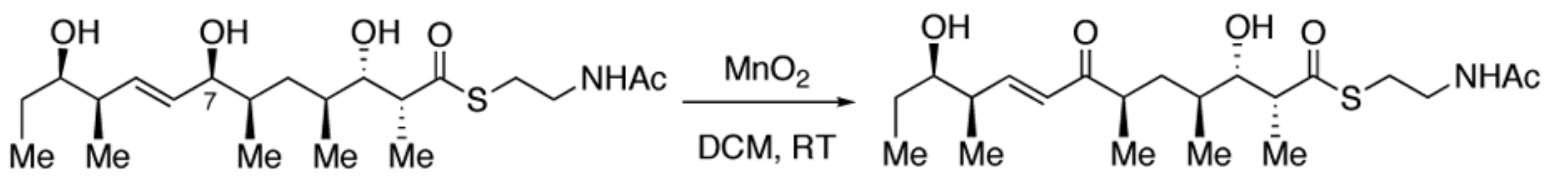
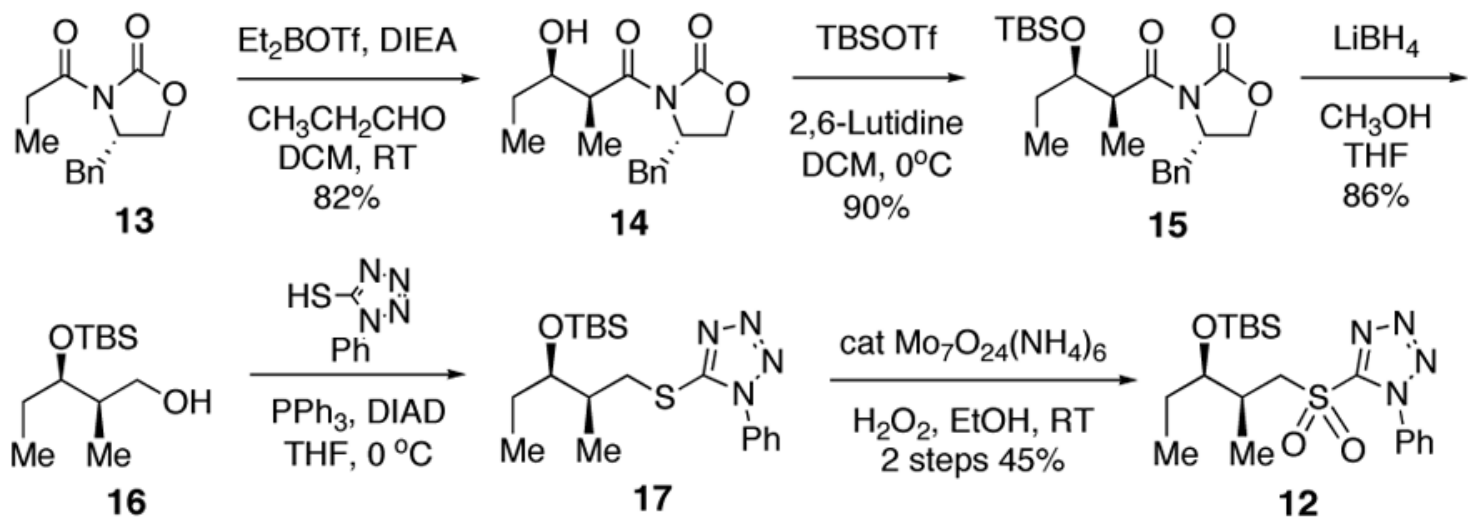

Scheme 2. 


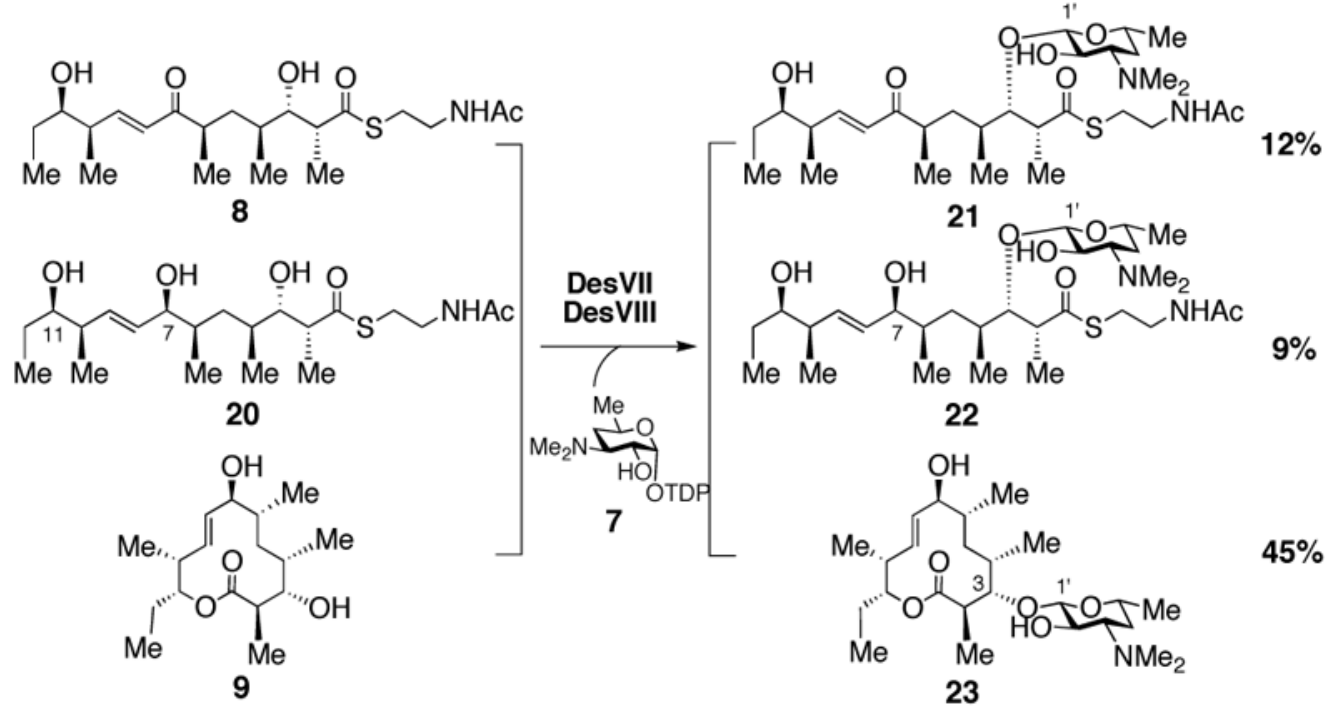

Scheme 3. 